\title{
A REMARK ON A RESULT DUE TO BLICHFELDT
}

\section{LOO-KENG HUA}

Let $\sigma \geqq 1$ and $\xi_{1}, \cdots, \xi_{n}$ be $n \geqq 3$ linear forms of the real variables $x_{1}, \cdots, x_{n}$ of nonvanishing determinant $\Delta$. For simplicity's sake we assume $|\Delta|=1$. Let $2 s$ of the forms be pairwise conjugate complex and the remaining $n-2 s$ be real. Then

$$
\left|\xi_{1}\right|^{\sigma}+\cdots+\left|\xi_{n}\right|^{\sigma} \leqq 1
$$

defines a symmetric convex body in the $x$-space, the volume $V(\sigma)$ of which equals

$$
2^{n} \cdot \frac{\{\Gamma(1+\alpha)\}^{n-2 s}\left\{\pi \Gamma(1+2 \alpha) / 2^{1+2 \alpha}\right\}:}{\Gamma(1+n \alpha)} \quad(\alpha=1 / \sigma) .
$$

Minkowski's principle states that there is a lattice point $\left(x_{1}, \cdots, x_{n}\right)$ $\neq(0, \cdots, 0)$ satisfying the inequality

$$
\left|\xi_{1}\right|^{\sigma}+\cdots+\left|\xi_{n}\right|^{\sigma} \leqq r^{\sigma}
$$

provided

$$
r^{n} \geqq 2^{n} V^{-1}(\sigma) .
$$

By means of Blichfeldt's method, van der Corput and Schaake ${ }^{1}$ obtained a sharpening of this result for $\sigma \geqq 2$. Decisive in this procedure is an inequality of the following form

$$
\sum_{p, q=1}^{k}\left|z_{p}-z_{q}\right|^{\sigma} \leqq \epsilon(\sigma) k \cdot \sum_{p=1}^{k}\left|z_{p}\right|^{\sigma},
$$

where the factor $\epsilon(\sigma)$ depends neither on the arbitrary complex numbers $z_{p}$ nor on $k$. Once such an inequality is known, (1) may be replaced by

$$
r^{n} \geqq(\epsilon(\sigma))^{n / \sigma} \cdot \frac{n+\sigma}{\sigma} \cdot V^{-1}(\sigma) .
$$

The elementary relation

$$
|u-v|^{\sigma} \leqq 2^{\sigma-1}\left(|u|^{\sigma}+|v|^{\sigma}\right)
$$

(following from the fact that $x^{\sigma}$ is a convex function of $x>0$ ) implies (2) with $\epsilon(\sigma)=2^{\sigma}$. Substituted in (3) this does not improve, but on

Received by the editors February 24, 1945.

1 Acta Arithmetica vol. 2 (1936) pp. 152-160. 
the contrary worsens, Minkowski's inequality. However, van der Corput and Schaake obtained the better value $2^{\sigma-1}$ for $\sigma \geqq 2$. I shall show here that $\epsilon(\sigma)=2$ is a legitimate choice for $1 \leqq \sigma \leqq 2$ and that both facts follow almost immediately from Marcel Riesz's convexity theorem.

Indeed, specialize this theorem (Theorem 296 on p. 219 of Hardy, Littlewood and Pólya's Inequalities) by taking $\gamma=\alpha$ and the $X$ as the linear forms $X_{p q}=z_{p}-z_{q}$. It then turns out that the logarithm of the maximum $M_{k}(\alpha)$ of

$$
\left\{\sum_{p, q=1}^{k}\left|z_{p}-z_{q}\right|^{1 / \alpha} / k \sum_{p=1}^{k}\left|z_{p}\right|^{1 / \alpha}\right\}^{\alpha}
$$

for fixed $k$ and variable $z_{1}, \cdots, z_{k}$ is a convex function of $\alpha$ in the interval $0 \leqq \alpha \leqq 1$. One readily verifies that

$$
M_{k}(0)=2, \quad M_{k}(1 / 2)=2^{1 / 2}, \quad M_{k}(1)=2(1-1 / k) \leqq 2 .
$$

As

$$
\left\{\sum_{p}\left|z_{p}\right|^{1 / \alpha}\right\}^{\alpha} \rightarrow \max \left|z_{p}\right| \text { for } \alpha \rightarrow 0
$$

the first equation follows from $\max \left|z_{p}-z_{q}\right| \leqq 2 \cdot \max \left|z_{p}\right|$ together with the observation that the upper bound 2 is attained for $z_{1}=1$, $z_{2}=-1, z_{3}=\cdots=z_{k}=0$. Similarly the two other equations are immediate consequences of the elementary inequalities

$$
\begin{gathered}
\sum_{p, q}\left|z_{p}-z_{q}\right|^{2}=2 k \sum_{p}\left|z_{p}\right|^{2}-2\left|\sum_{p} z_{p}\right|^{2} \leqq 2 k \sum_{p}\left|z_{p}\right|^{2}, \\
\sum_{p \neq q}\left|z_{p}-z_{q}\right| \leqq \sum_{p \neq q}\left(\left|z_{p}\right|+\left|z_{q}\right|\right)=2(k-1) \sum_{p}\left|z_{p}\right|,
\end{gathered}
$$

and the corresponding obvious observations about the $z_{p}$ for which the upper bound is reached.

Let us use 2 as the basis of our logarithms. Then the values of $\log _{2} M_{k}(\alpha)$ are $1,1 / 2$ and less than or equal to 1 for $\alpha=0,1 / 2,1$ respectively, and hence the broken line consisting of $1-\alpha$ for $0 \leqq \alpha \leqq 1 / 2$ and $\alpha$ for $1 / 2 \leqq \alpha \leqq 1$ gives an upper bound for the convex function $\log _{2} M_{k}(\alpha)$. We thus obtain the promised result that (2) holds with

$$
\epsilon(\sigma)=2^{\sigma-1} \text { for } \sigma \geqq 2 \text { and } \epsilon(\sigma)=2 \text { for } 1 \leqq \sigma \leqq 2 .
$$

Both choices are the best possible of their kinds, as, for $0 \leqq \alpha \leqq 1 / 2$, is shown by the example $k=2, z_{1}=-z_{2}=1$, and, for $1 / 2 \leqq \alpha \leqq 1$, by the example $z_{1}=-z_{2}=1, z_{3}=\cdots=z_{k}=0$, with large $k$. 
Consider the case $1 \leqq \sigma \leqq 2$. If we substitute the value $\epsilon(\sigma)=2$ in (3), we shall find that it does not always improve Blichfeldt's known inequality, in particular not for the most interesting case $\sigma=1$. We observe that

$$
\left(\frac{\left|\xi_{1}\right|^{\sigma}+\cdots+\left|\xi_{n}\right|^{\sigma}}{n}\right)^{1 / \sigma}
$$

is an increasing function of the exponent $\sigma$, while the upper bound for its lattice minimum as derived from (3), namely,

$$
\left(\frac{2}{n}\right)^{1 / \sigma}\left(\frac{n+\sigma}{\sigma}\right)^{1 / n}(V(\sigma))^{-1 / n}
$$

is not. For $s=0$ the expression (5) tends to a limit with $n \rightarrow \infty$, namely

$$
\frac{1}{2}\left(\frac{2}{\sigma e}\right)^{1 / \sigma} / \Gamma\left(1+\frac{1}{\sigma}\right)=2^{\alpha-1}\left(\frac{\alpha}{e}\right)^{\alpha} / \Gamma(1+\alpha) .
$$

The logarithmic derivative of this function with respect to $\alpha$ is negative for $\alpha=1 / 2$ and positive for $\alpha=2 / 3$, and hence this function has a minimum between $\sigma=2$ and $\sigma=1.5$; numerical computation gives as its location $\sigma=\sigma_{0}=1.8653 \cdots .^{2}$ At this point the value of the function is

$$
\leqq 1 /(3.146 e)^{1 / 2}
$$

which is slightly better than the constant

due to Blichfeldt. ${ }^{3}$

$$
1 /(\pi e)^{1 / 2}
$$

In conclusion, for $2 \geqq \sigma \geqq \sigma_{0}$, (1) may be replaced by

$$
r^{n} \geqq 2^{n / \sigma}\left(\frac{n+\sigma}{\sigma}\right) V^{-1}(\sigma),
$$

and, for $1 \leqq \sigma \leqq \sigma_{0}$, (1) may be replaced by

$$
r^{n} \geqq 2^{n / \sigma_{0}}\left(\frac{n+\sigma_{0}}{\sigma_{0}}\right) V^{-1}\left(\sigma_{0}\right) .
$$

This would be true however $\sigma_{0}$ were chosen within the limits $1 \leqq \sigma_{0} \leqq 2$; our special choice approaches the best possible for $n \rightarrow \infty$ (and $s=0$ ) and is sharp enough to beat Blichfeldt's record by a slight margin, even for small $n$.

\section{National Tsing Hua University}

\footnotetext{
2 The author is indebted to Mr. Sze for this numerical value.

${ }^{3}$ Trans. Amer. Math. Soc. vol. 15 (1914) pp. 227-235.
} 